\title{
Protective effects of saffron against zearalenone- induced alterations in reproductive hormones in female mice (Mus musculus)
}

\author{
Bashir Ahmad' , Vinoy K. Shrivastava' ${ }^{1}$, Ramadan Saleh², Ralf Henkel ${ }^{3}$, Ashok Agarwal ${ }^{4}$ \\ 'Laboratory of Endocrinology, Department of Bioscience, Barkatullah University, Bhopal, India; ${ }^{2}$ Department of Dermatology, Venereology and \\ Andrology, Faculty of Medicine, Sohag University, Sohag, Egypt; ${ }^{3}$ Department of Medical Bioscience, University of the Western Cape, Bellville, South \\ Africa; ${ }^{4}$ American Center for Reproductive Medicine, Urological Institute, Cleveland Clinic, Cleveland, $\mathrm{OH}$, USA
}

Objective: Zearalenone (ZEA) is a mycotoxin with potent estrogenic effects. Saffron is an herbal product that has antioxidant activities. The objective of this study was to investigate the protective role of saffron against reproductive toxicity induced by ZEA in female mice.

Methods: Ninety 8-week-old female mice were randomly allocated into three treatment groups. The first group received an intraperitoneal injection of ZEA ( $2.5 \mathrm{mg} / \mathrm{kg})$ on alternate days. The second group received ZEA $(2.5 \mathrm{mg} / \mathrm{kg})$ on alternate days plus oral saffron daily $(50 \mathrm{mg} / \mathrm{kg})$. The third group was treated with a vehicle of $1 \%$ dimethyl sulfoxide (DMSO) on alternate days, as a control. Ten mice were euthanized from each group at 30,60, and 90 days of treatment. Serum levels of luteinizing hormone ( $\mathrm{LH})$, follicle-stimulating hormone (FSH), estradiol $\left(\mathrm{E}_{2}\right)$, and progesterone $(\mathrm{P})$ were assessed. The uterus and ovaries were examined for changes in size or morphology.

Results: Serum levels of $\mathrm{LH}, \mathrm{FSH}, \mathrm{E}_{2}$, and $\mathrm{P}$ in the female mice treated with ZEA plus saffron were significantly higher than in those treated with ZEA alone, and were not significantly different from those treated with $1 \%$ DMSO. The female mice treated with ZEA alone showed a reduction in size of the uterus and abnormal architecture of the ovaries.

Conclusion: The administration of saffron to female mice resulted in a significant reduction in ZEA-induced alterations in reproductive hormone levels, the size of the uterus, and the morphology of the ovaries.

Keywords: Antioxidants; Mus musculus female mice; Mycotoxicity; Reproductive hormones; Saffron; Zearalenone

\section{Introduction}

An endocrine disruptor is an exogenous substance or mixture that alters the functions of the endocrine system and consequently causes adverse health effects in an intact organ, its progeny, or subpopulation [1]. Close to 800 chemicals are known or suspected to be capable of interfering with hormone receptors, hormone synthesis, or hormone conversion in mammals. Zearalenone (ZEA) is a nonste-

Received: May 6, 2018 · Revised: Oct 2, 2018 · Accepted: Oct 16, 2018

Corresponding author: Ramadan Saleh

Department of Dermatology, Venereology and Andrology, Faculty of Medicine, Sohag University, Sohag 82524, Egypt

Tel:+20-093-2304772 Fax:+20-093-2304773 E-mail:salehr2010@yahoo.com

This is an Open Access article distributed under the terms of the Creative Commons Attribution Non-Commercial License (http://creativecommons.org/licenses/by-nc/4.0/) which permits unrestricted non-commercial use, distribution, and reproduction in any medium, provided the original work is properly cited. roidal, potent, estrogenic mycotoxin produced by fungi belonging to the genus Fusarium in foods and feeds [2]. It has been implicated in reproductive disorders of farm animals and hyperestrogenic syndromes in humans $[3,4]$.

The most studied mechanism of action of ZEA and other phytoestrogens involves their ability to bind to $\alpha$ - and $\beta$-estrogen receptors [5-8]. The toxin produces agonistic and antagonistic effects on the estrogen receptor for $17 \beta$-estradiol, and thus exhibits distinct estrogenic properties, with varying effects on the reproductive system in several species of animals [9]. Other suggested mechanisms of action of ZEA include interactions with transport proteins and the cell membrane [10,11], as well as tissue oxidative stress [12].

The latter mechanisms indicate that ZEA may play a role in oncogenesis through cytotoxicity, immunotoxicity, DNA damage, and chromosomal aberration in rodents and humans $[13,14]$. The clinical 
signs observed in female animals in response to phytoestrogens include enlargement of the mammary glands, swelling of the vulva, rectal-vaginal prolapse, ovarian and uterine impairment, and hormonal imbalances $[15,16]$. In addition, ZEA has been shown to dramatically reduce the number and motility of live spermatozoa in adult male albino mice $[17,18]$.

Proposed ways to reduce mycotoxicity in animals and humans involve the use of many vitamins, minerals, nutrients, and herbal products. Saffron is a widely-used herbal remedy that has a unique chemical composition of more than 150 volatile compounds and several nonvolatile compounds. Commercial saffron is produced from dried stigmas of Crocus sativus, a member of the large family Iridaceae that is cultivated in Azerbaijan, France, Greece, India (Kashmir), Iran, Italy, Spain, China, Morocco, Turkey, Egypt, and Mexico. The main components of saffron, such as crocin and saffranal, have a wide range of beneficial biological activities with no toxic side effects [19].

The medical properties of saffron have been attributed to its potent antioxidant activity due to the presence of crocin, a unique carotenoid $[20,21]$. It may enhance pituitary-ovarian axis activity; boost levels of luteinizing hormone (LH), follicle-stimulating hormone (FSH) and estradiol $\left(\mathrm{E}_{2}\right)$; and stimulate folliculogenesis in human females [22]. In males, saffron has been shown to rejuvenate the reproductive system, which may help ameliorate conditions such as erectile dysfunction, premature ejaculation, low sperm count, and low sperm motility [23]. The objective of this study was to investigate the protective role of saffron against reproductive toxicity induced by ZEA in female mice.

\section{Methods}

The study was approved by the Ethical Committee of Barkatullah University, Bhopal, India. The experiments were carried out in accordance with the U.K. Animals (Scientific Procedures) Act, 1986 and associated guidelines, as well as the EU Directive 2010/63/EU for animal experiments.

\section{Reagents}

ZEA was obtained in a powder form (no. Z2125; Sigma Aldrich, Bhopal, India). It was weighed, under completely aseptic conditions, using a digital scale with $0.01 \mathrm{~g}$ precision, and then dissolve in $1 \%$ dimethyl sulfoxide (DMSO) to form stock solutions that were stored in a refrigerator at $4^{\circ} \mathrm{C}$ until use. The crude form of saffron was purchased from Sigma (Pulwama, India). The required amount of saffron was dissolved in drinking water for oral administration. Both chemicals were identified and verified by the Department of Food Technology, Islamic University (Kashmir, India).

\section{Experimental animals}

The study included 90 clinically healthy, 8-week-old, adult female mice (Parkes strain of Mus musculus species), with an initial body weight of $25 \pm 5 \mathrm{~g}$. The mice were provided by the Animal Laboratory of Reproductive Endocrinology Unit, Department of Bioscience, Barkatullah University, Bhopal, India. All mice were fed daily with equal amounts of a balanced oral diet at regular intervals.

\section{Animal groups and treatment}

The mice were randomly divided into three treatment groups (30 mice each). The first group received an intraperitoneal injection of ZEA $(2.5 \mathrm{mg} / \mathrm{kg}$ ) on alternate days. The second group received an intraperitoneal injection of $2.5 \mathrm{mg} / \mathrm{kg}$ of ZEA on alternate days, as well as a daily dose of oral saffron (50 mg/kg with drinking water). The third group received an intraperitoneal injection of $1 \%$ DMSO vehicle on alternate days, and served as a control. Mice were observed for remarkable side effects or complications.

\section{Assessment of reproductive hormones}

Ten mice were euthanized from each group after 30,60, and 90 days of treatment. A serum reproductive hormone assay was conducted as previously described [24]. Briefly, a morning venous blood sample was drawn, in a proestrus period, with 27-gauge needles, through a 1-mL syringe into ethylenediaminetetraacetic acid (EDTA) heparinized containers. Levels of $\mathrm{LH}, \mathrm{FSH}, \mathrm{E}_{2}$ and progesterone (P) were assessed in serum samples by an automated quantitative test using an enzyme-linked immunosorbent assay kit according to the manufacturer's guidelines (Thermo Multiskan Lab System, Champaign, IL, USA). The resulting levels of serum $\mathrm{LH}, \mathrm{FSH}, \mathrm{E}_{2}$, and $\mathrm{P}$ were expressed as $\mathrm{IU} / \mathrm{mL}$, IU/mL, $\mathrm{ng} / \mathrm{mL}$, and $\mathrm{ng} / \mathrm{mL}$, respectively.

\section{Evaluation of gross morphology of the uterus and ovaries}

The size and gross morphology of the uterus and ovaries of the mice euthanized after 90 days of treatment were recorded, and photographs were taken.

\section{Histological evaluation of the ovaries}

The ovaries were dissected from the mice sacrificed at the different treatment intervals (i.e., 30, 60, and 90 days) for a histological evaluation. The ovaries were washed thoroughly in $0.85 \%$ physiological saline $(\mathrm{NaCl})$ and preserved in aqueous Bouin solution. The ovarian tissue sections were processed with an automated tissue processor and embedded in paraffin. Ovarian tissue sections measuring $5 \mu \mathrm{m}$ in thickness were cut with a rotary microtome and stained with hematoxylin and eosin. The histological sections were examined using light microscopy under a magnification of $\times 400$, and digital images were taken in the microphotography unit. 


\section{Statistical analysis}

The data were analyzed using SPSS ver. 17.0 (SPSS Inc., Chicago, IL, USA). Results of the experiments were expressed as mean and the standard error of the mean. The differences between the mean values were evaluated by one-way analysis of variance. Statistical significance was considered at $p$-values $<0.05$.

\section{Results}

Table 1 presents comparisons of serum levels of $\mathrm{LH}, \mathrm{FSH}, \mathrm{E}_{2}$, and $\mathrm{P}$ in the female mice that received $1 \%$ DMSO vehicle (control group), ZEA alone, and ZEA plus saffron after 30,60, and 90 days of administration. The gross morphological features of the uterus and ovaries of the fe-

Table 1. Comparisons of serum levels of $\mathrm{LH}, \mathrm{FSH}, \mathrm{E}_{2}$, and $\mathrm{P}$ in female mice receiving $1 \%$ DMSO vehicle (placebo), ZEA alone, and ZEA plus saffron after 30,60 , and 90 days of administration

\begin{tabular}{|c|c|c|c|c|}
\hline \multirow{2}{*}{ Variable } & \multirow{2}{*}{ Group $(n=10)$} & \multicolumn{3}{|c|}{ Duration } \\
\hline & & 30 Days & 60 Days & 90 Days \\
\hline \multirow[t]{3}{*}{$\mathrm{LH}(\mathrm{IU} / \mathrm{mL})$} & 1\% DMSO & $6.14 \pm 0.04$ & $6.64 \pm 0.09$ & $6.67 \pm 0.06$ \\
\hline & ZEA alone & $4.60 \pm 0.07^{a)}$ & $4.97 \pm 0.00^{\mathrm{a})}$ & $4.94 \pm 0.06^{\mathrm{a})}$ \\
\hline & ZEA+saffron & $5.90 \pm 0.01^{c)}$ & $6.44 \pm 0.02$ (NS) & $6.47 \pm 0.02$ (NS) \\
\hline \multirow[t]{3}{*}{ FSH (IU/mL) } & 1\% DMSO & $10.61 \pm 0.01$ & $10.64 \pm 0.09$ & $10.57 \pm 0.01$ \\
\hline & ZEA alone & $6.46 \pm 0.02^{\mathrm{a})}$ & $5.07 \pm 0.09^{\mathrm{a})}$ & $5.05 \pm 0.54^{\mathrm{a})}$ \\
\hline & ZEA+saffron & $8.13 \pm 0.01^{\mathrm{b})}$ & $9.73 \pm 0.02$ (NS) & $9.85 \pm 0.00(\mathrm{NS})$ \\
\hline \multirow[t]{3}{*}{$\mathrm{E}_{2}(\mathrm{ng} / \mathrm{mL})$} & 1\% DMSO & $20.12 \pm 0.03$ & $21.45 \pm 0.17$ & $22.16 \pm 0.12$ \\
\hline & ZEA alone & $16.57 \pm 0.09^{a)}$ & $13.73 \pm 0.11^{\text {a) }}$ & $13.33 \pm 0.14^{a)}$ \\
\hline & ZEA+saffron & $18.01 \pm 0.01^{b)}$ & $20.67 \pm 0.15$ (NS) & $21.78 \pm 0.78(\mathrm{NS})$ \\
\hline \multirow[t]{3}{*}{$P(n g / m L)$} & $1 \%$ DMSO & $1.88 \pm 0.08$ & $1.94 \pm 0.01$ & $1.95 \pm 0.06$ \\
\hline & ZEA alone & $1.42 \pm 0.00^{c)}$ & $1.71 \pm 0.01^{c)}$ & $1.68 \pm 0.03^{c)}$ \\
\hline & ZEA+saffron & $1.64 \pm 0.06$ (NS) & $1.89 \pm 0.04$ (NS) & $1.89 \pm 0.06(\mathrm{NS})$ \\
\hline
\end{tabular}

Values are presented as mean \pm standard error. One-way analysis of variance was used for the statistical analysis.

$\mathrm{LH}$, luteinizing hormone; $\mathrm{FSH}$, follicle-stimulating hormone; $\mathrm{E}_{2}$, estradiol; P, progesterone; DMSO, dimethyl sulfoxide; ZEA, zearalenone; NS, not significant. a) $p<0.001 ;{ }^{\text {b) }} p<0.01 ;{ }^{c} p<0.05$.
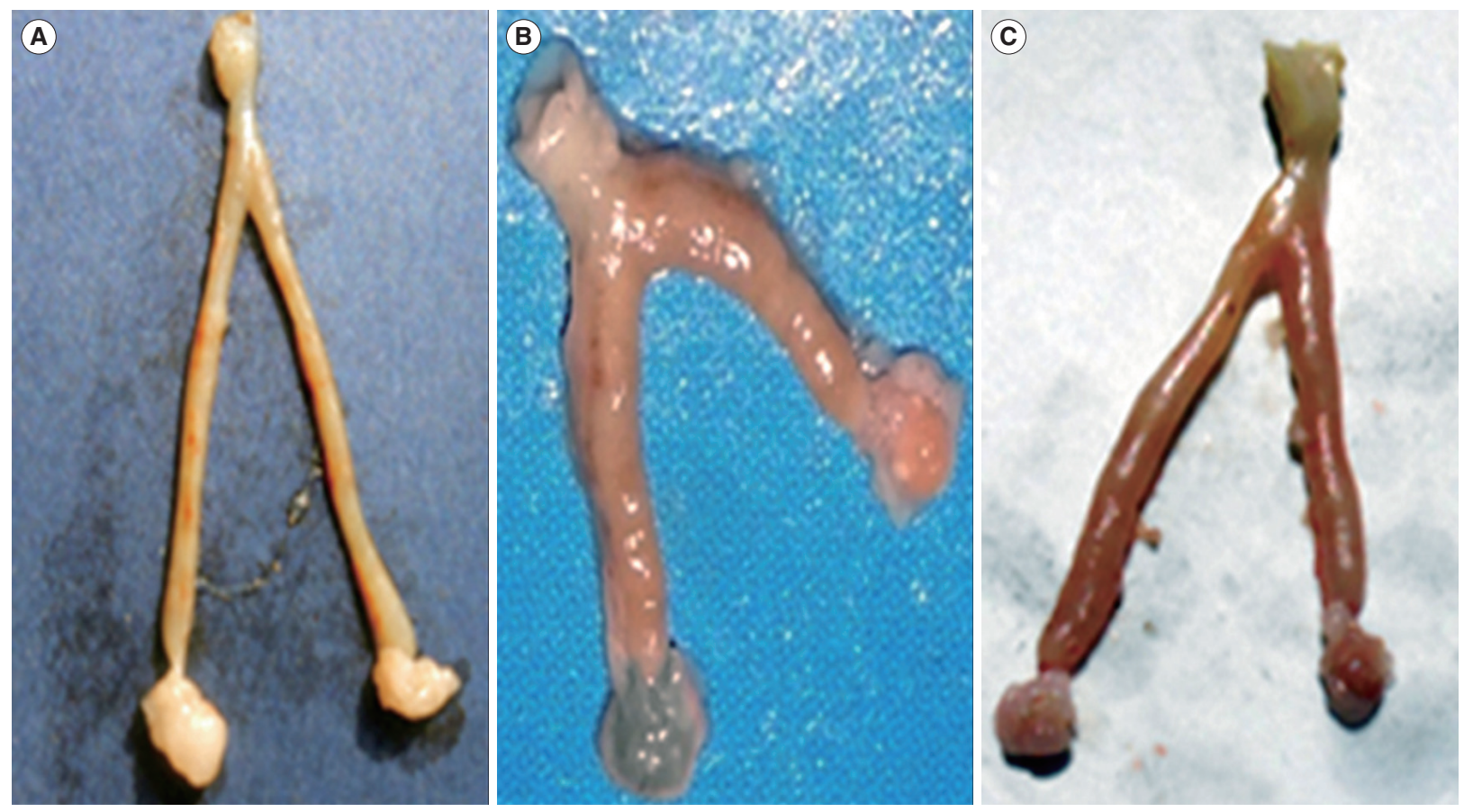

Figure 1. Gross morphological features of the uterus and ovaries in female mice after 90 days of treatment with (A) $1 \%$ dimethyl sulfoxide (DMSO), (B) zearalenone (ZEA) alone, and (C) ZEA plus saffron. Female mice treated with 1\% DMSO showed normal appearance of the uterus and ovaries. Female mice treated with ZEA alone showed thickening, swelling, and shortening of the uterus and ovaries. Female mice treated with ZEA plus saffron exhibited congested but less abnormal features of the uterus and ovaries compared to the group treated with ZEA alone. 

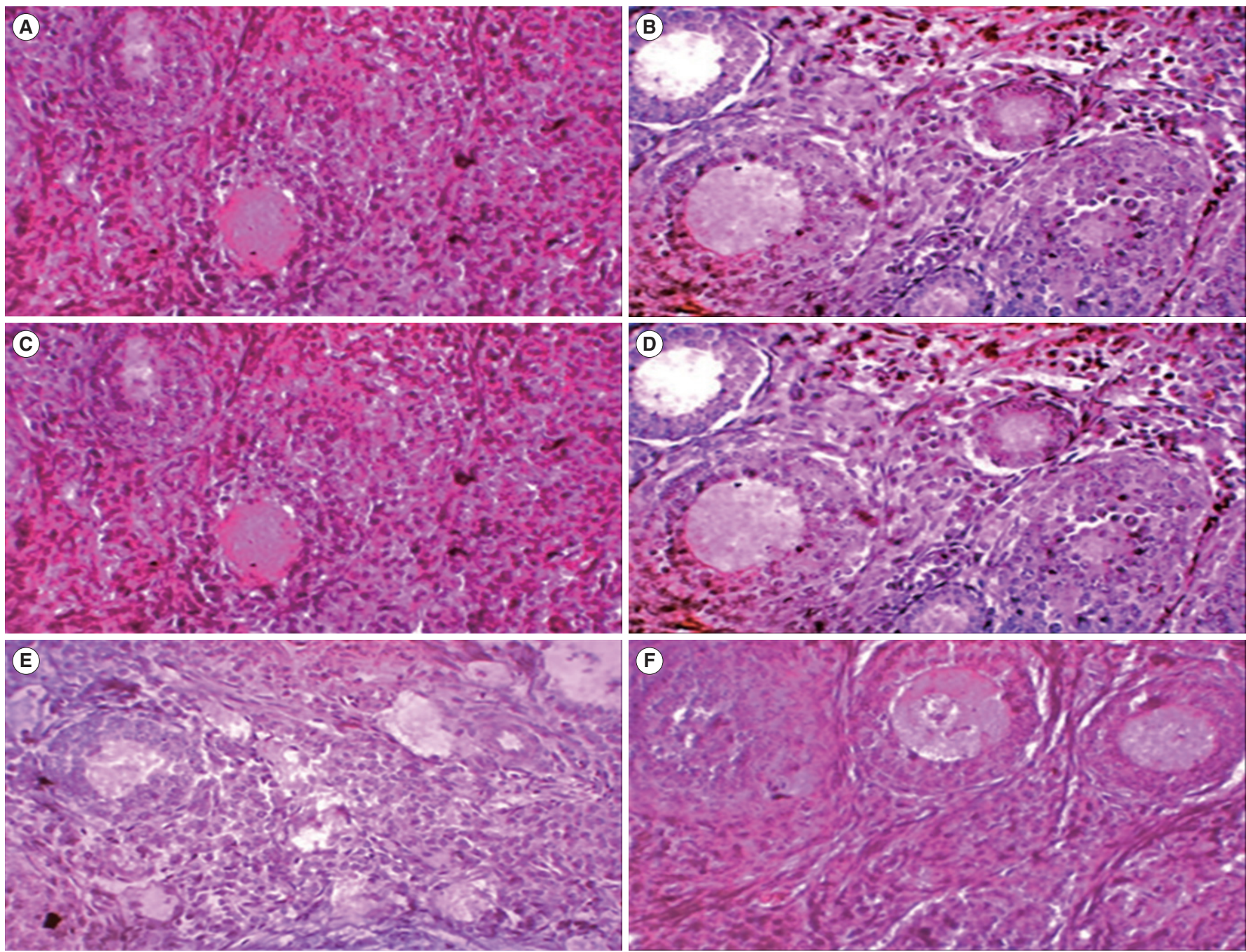

Figure 2. Microscopic pictures of mouse ovarian tissue $(H \& E, \times 400)$. Representative images of $H \& E$ staining of ovarian tissue from adult female mice after treatment with zearalenone (ZEA) alone for 30 days (A), for 60 days (C), and for 90 days (E); and ZEA plus saffron for 30 days (B), for 60 days (D), and for 90 days (F).

male mice after 90 days of treatment with 1\% DMSO vehicle (control), ZEA alone, and ZEA plus saffron are shown in Figure 1. Female mice treated with ZEA alone showed a reduced size of the uterus and an abnormal architecture of the ovaries, while those treated with $1 \%$ DMSO vehicle, or ZEA plus saffron showed no remarkable abnormalities in the size and gross morphology of the uterus or ovaries.

A reduction of the regular food intake capacity was observed in the group of mice treated with ZEA alone. As a result, the body weight (g) was significantly reduced $(p<0.01)$ in the female mice treated with ZEA alone at 30 days $(24.3 \pm 0.6), 60$ days ( $23.3 \pm 0.9)$, and 90 days $(21.1 \pm 0.8)$ compared to the base-line values of body weight within the same group $(27.2 \pm 0.5,27.9 \pm 0.6$, and $28.1 \pm 0.9$, respectively). The effects of the interventions with ZEA alone and ZEA plus saffron on the histological features of the ovaries of adult female mice after 30, 60, and 90 days are shown in Figure 2.
Adult female mice treated with ZEA alone for 30 days showed a reduction in the number of large and mature follicles, loosely arranged follicular cells, atretic follicles, and a lack of corpus luteum, with signs of vacuolization and degeneration of the connective tissue stroma in the medulla (Figure $2 \mathrm{~A}$ ). Those features were aggravated in adult female mice treated with ZEA alone for 60 and 90 days (Figure $2 \mathrm{C}, 2 \mathrm{E}$, respectively). Adult female mice treated with ZEA plus saffron for 30 , 60 , and 90 days showed normal-looking follicles with proper lining of granulosa cells, organized corpus luteum, and less degenerative change in the medullary region (Figure $2 \mathrm{~B}, 2 \mathrm{D}$, and $2 \mathrm{~F}$, respectively).

\section{Discussion}

A healthy hypothalamic-pituitary-gonadal axis is essential for mammalian reproduction. A large number of compounds that are 
continuously released into the environment can mimic the action of endogenous estrogen, possibly disrupting endocrine function in mammals [10]. However, only a small fraction of those chemicals have been investigated through tests capable of identifying overt endocrine effects in intact organisms [1]. Toxicants that interfere with ovarian function can affect the ovary directly or indirectly by acting at the level of hypothalamus, pituitary gland, or both [25].

In this study, administration of ZEA alone resulted in a significant reduction in the serum levels of reproductive pituitary (LH and FSH) and ovarian ( $E_{2}$ and $P$ ) hormones in female mice, which could be observed at multiple treatment intervals (30,60, and 90 days). Interestingly, a further reduction of serum levels of $E_{2}$ was observed in the mice treated with ZEA alone when the treatment duration was extended to 60 and 90 days. A previous study reported decreased levels of circulating $E_{2}$ and $P$ in response to various concentrations of ZEA $(2,4$, and $8 \mathrm{mg} / \mathrm{kg})$ [26].

However, the authors of that study indicated that serum levels of LH or FSH were not affected by ZEA treatment. The lack of significant differences in LH and FSH was attributed to the pregnant status of the animals included in that study. In contrast, in another study, both $\mathrm{LH}$ and FSH slightly increased in response to a dose of $8 \mathrm{mg} / \mathrm{kg}$ of ZEA [27].

The exact mechanisms through which reproductive hormone levels are altered in female mice subjected to ZEA treatment remain unclear. A study suggested that ZEA may exert an inhibitory action at the level of the pituitary, rather than affecting the hypothalamus [28]. It may be speculated that reduced levels of FSH inhibit follicular development, thereby preventing increases in $\mathrm{E}_{2}$ and $\mathrm{P}$ levels. The lack of $E_{2}$ and $P$ positive feedback on $\mathrm{LH}$ release may then prevent ovulation. It has been reported that chemicals can delay or block ovulation by disrupting the ovulatory surge of $\mathrm{LH}$, or by interfering with the ability of maturing follicles to respond to gonadotropic signals [29].

The results of this study indicated that serum levels of $L H, F S H, E_{2}$, and $\mathrm{P}$ in the group of female mice treated with ZEA plus saffron were significantly higher than levels in the mice treated with ZEA alone, and were not significantly different from those in the mice treated with 1\% DMSO (placebo). In addition, levels of reproductive hormones in the groups of mice treated with ZEA plus saffron or placebo did not show significant alterations at different treatment intervals. Therefore, the coadministration of saffron with ZEA in female mice resulted in hormone levels comparable to those obtained from treatment with placebo.

The results of this study indicated significant impairments in the histological features of adult female mice ovaries treated with ZEA alone for 30 days. Extending the treatment periods for 60 and 90 days resulted in additional detrimental effects on ovarian histology. Therefore, the potential reproductive toxicity induced by ZEA may be dose-dependent and may accumulate over time. Interestingly, female mice treated with ZEA plus saffron for the same periods of time did not show histopathological abnormalities similar to those that appeared in the mice treated with ZEA alone. The latter finding is consistent with lack of gross morphological abnormalities of the ovaries of female mice treated with ZEA plus saffron.

These results suggest that saffron may play a role in antagonizing the negative effects of ZEA on the serum levels of reproductive hormones in female mice and on ovarian and uterine morphology. However, the exact mechanisms by which saffron exerts its positive protective effects against ZEA-induced reproductive toxicity remain unclear. Nonetheless, a growing body of evidence on the potential therapeutic effects of saffron has emerged over the last two decades. A previous study observed reductions in the metabolic and behavioral signs of stress in saffron-treated rodents [30]. In a recent study, fasting blood sugar (FBS) levels decreased significantly and reached normoglycemic levels in diabetic male rats treated with saffron [31]. The reduction of FBS in that study was linked to increased serum levels of adiponectin, a protein hormone that is secreted by adipocytes and plays an important role in regulating the glucose and fatty acid metabolic pathways. Similarly, the consumption of saffron combined with resistance exercise in diabetic rats had strong therapeutic effects on diabetic parameters both in vivo and in vitro [32]. However, the antidiabetic properties of saffron, in the latter study, were attributed to a redox-mediated mechanism affecting insulin secretion and glucose uptake.

An experimental study indicated that gonadotropin-releasing hormone and gonadotropic hormones were released by the activation of noradrenergic nerves in the locus ceruleus in response to crocin, which is contained in saffron [22]. Another study suggested that crocin and crocetin may play a role in stimulating the activity of serotonergic nerves, which was related to their antidepressant properties [33]. More recently, a study indicated that the administration of an alcoholic extract of saffron reduced FSH levels in the blood of 12-month-old rats to a level close to the FSH levels reported in 6-month-old rats [34]. Moreover, the authors of that study reported an increase in the number and body weight of pups from rats that received the saffron extract prior to pairing with intact males.

These results may have important implications, particularly in light of the growing concern on the declining fertility of mammals in response to the large number of endocrine-disrupting compounds that are being continuously released into the environment. In 2013, the United Nations Environment Programme and the World Health Organization published a report stating that the effects of endocrinedisrupting compounds are endocrine system-related and not necessarily species dependent [1]. Mice are an established model for studying the effects of agents on humans as they are easy and flexi- 
ble to handle and manipulate [35]. Similarities exist between mouse and human reproductive organs and cycles [36], enabling the careful extrapolation of findings to humans. Future efforts should primarily focus on implementing effective strategies to reduce mammalian exposure to endocrine-disrupting compounds.

In conclusion, the exposure of female mice to ZEA resulted in alterations in reproductive hormones, reduced uterus size, and an abnormal architecture of the ovaries. Coadministration of saffron with ZEA resulted in amelioration of ZEA-induced reproductive toxicity. These observations may be related, at least in part, to the antioxidant properties of saffron. However, future studies are warranted to understand the exact mechanisms of the positive protective effects of saffron against the reproductive damage caused by endocrine-disrupting compounds.

\section{Conflict of interest}

No potential conflict of interest relevant to this article was reported.

\section{Acknowledgments}

The authors would like to thank Professor Hossein Hosseinzadeh, $\mathrm{PhD}$, Pharmaceutical Research Center, Department of Pharmaco-dynamics and Toxicology, School of Pharmacy, Mashhad University; and Professor Zahra Bathaie, PhD, Department of Clinical Biochemistry, Tarbiat Modares University, for their help with conducting the experiments.

\section{References}

1. Bergman A, Heindel JJ, Jobling S, Kidd KA, Zoeller RT. State of the science of endocrine disrupting chemicals 2012: summary for decision-makers. Geneva: World Health Organization; 2013.

2. Escriva $L$, Font $G$, Manyes $L$. In vivo toxicity studies of Fusarium mycotoxins in the last decade: a review. Food Chem Toxicol 2015;78:185-206.

3. Gallo D, Cantelmo F, Distefano M, Ferlini C, Zannoni GF, Riva A, et al. Reproductive effects of dietary soy in female Wistar rats. Food Chem Toxicol 1999;37:493-502.

4. Rosselli M, Reinhart K, Imthurn B, Keller PJ, Dubey RK. Cellular and biochemical mechanisms by which environmental oestrogens influence reproductive function. Hum Reprod Update 2000;6:332-50.

5. Whitten PL, Patisaul HB. Cross-species and interassay comparisons of phytoestrogen action. Environ Health Perspect 2001;109 Suppl 1:5-20.

6. Tapiero H, Ba GN, Tew KD. Estrogens and environmental estro- gens. Biomed Pharmacother 2002;56:36-44.

7. Malekinejad H, Maas-Bakker RF, Fink-Gremmels J. Bioactivation of zearalenone by porcine hepatic biotransformation. Vet Res 2005;36:799-810.

8. Dusza L, Ciereszko R, Skarzynski DJ, Nogowski L, Opalka M, Kaminska B, et al. Mechanism of phytoestrogens action in reproductive processes of mammals and birds. Reprod Biol 2006;6 Suppl 1:151-74.

9. Abbes S, Salah-Abbes JB, Ouanes Z, Houas Z, Othman O, Bacha H, et al. Preventive role of phyllosilicate clay on the immunological and biochemical toxicity of zearalenone in Balb/c mice. Int Immunopharmacol 2006;6:1251-8.

10. Benassayag C, Perrot-Applanat M, Ferre F. Phytoestrogens as modulators of steroid action in target cells. J Chromatogr B Analyt Technol Biomed Life Sci 2002;777:233-48.

11. Poor M, Kunsagi-Mate S, Balint M, Hetenyi C, Gerner Z, Lemli B. Interaction of mycotoxin zearalenone with human serum albumin. J Photochem Photobiol B 2017;170:16-24.

12. Hou YJ, Zhao YY, Xiong B, Cui XS, Kim NH, Xu YX, et al. Mycotoxincontaining diet causes oxidative stress in the mouse. PLoS One 2013;8:e60374.

13. National Toxicology Program. Carcinogenesis bioassay of zearalenone (CAS No. 17924-92-4) in F344/N rats and B6C3F1 mice (FEED study). Natl Toxicol Program Tech Rep Ser 1982;235:1-155.

14. Zinedine A, Soriano JM, Molto JC, Manes J. Review on the toxicity, occurrence, metabolism, detoxification, regulations and intake of zearalenone: an oestrogenic mycotoxin. Food Chem Toxicol 2007;45:1-18.

15. Ahmad B, Chouhan S, Shrivastava VK. Effect of zearalenone (Mycoestrogen) on morphometrics of female mice and ameliorative role of saffron. Egypt Acad J Biol Sci B Zool 2015;7:1-12.

16. Nakamura U, Rudolf FO, Pandey K, Kadokawa H. The non-steroidal mycoestrogen zeranol suppresses luteinizing hormone secretion from the anterior pituitary of cattle via the estradiol receptor GPR30 in a rapid, non-genomic manner. Anim Reprod Sci 2015;156:118-27.

17. Kim IH, Son HY, Cho SW, Ha CS, Kang BH. Zearalenone induces male germ cell apoptosis in rats. Toxicol Lett 2003;138:185-92.

18. Sambuu R, Takagi M, Namula Z, Nii M, Taniguchi M, Uno S, et al. Effects of long-term in vitro exposure of ejaculated boar sperm to zearalenone and a-zearalenol in sperm liquid storage medium. Anim Sci J 2013;84:28-34.

19. Asai A, Nakano T, Takahashi M, Nagao A. Orally administered crocetin and crocins are absorbed into blood plasma as crocetin and its glucuronide conjugates in mice. J Agric Food Chem 2005; 53:7302-6.

20. Giaccio M. Crocetin from saffron: an active component of an an- 
cient spice. Crit Rev Food Sci Nutr 2004;44:155-72.

21. Yaribeygi H, Mohammadi MT, Sahebkar A. Crocin potentiates antioxidant defense system and improves oxidative damage in liver tissue in diabetic rats. Biomed Pharmacother 2018;98:3337.

22. Mokhtari M, Sharifi E, Daneshi A. Effects of hydro-alcoholic extract of red dried stigmas of Crocus sativus L. flowers (saffron) on the levels of pituitary-ovary hormones and folliculogenesis in rats. Int J Fertil Steril 2010;3:185-90.

23. Szafranska B, Ziecik A, Okrasa S. Primary antisera against selected steroids or proteins and secondary antisera against gammaglobulins: an available tool for studies of reproductive processes. Reprod Biol 2002;2:187-204.

24. Wu J, Shao S, Zhou F, Wen S, Chen F, Han X. Reproductive toxicity on female mice induced by microcystin-LR. Environ Toxicol Pharmacol 2014;37:1-6.

25. Guney M, Demirin H, Oral B, Ozguner M, Bayhan G, Altuntas I. Ovarian toxicity in rats caused by methidathion and ameliorating effect of vitamins $E$ and C. Hum Exp Toxicol 2007;26:491-8.

26. Milano GD, Becu-Villalobos D, Tapia MO. Effects of long-term zearalenone administration on spermatogenesis and serum luteinizing hormone, follicle-stimulating hormone, and prolactin values in male rats. Am J Vet Res 1995;56:954-8.

27. Collins TF, Sprando RL, Black TN, Olejnik N, Eppley RM, Alam HZ, et al. Effects of zearalenone on in utero development in rats. Food Chem Toxicol 2006;44:1455-65.

28. Whitten PL, Naftolin F. Reproductive actions of phytoestrogens. Baillieres Clin Endocrinol Metab 1998;12:667-90.

29. Goldman JM, Cooper RL, Edwards TL, Rehnberg GL, McElroy WK, Hein JF. Suppression of the luteinizing hormone surge by chlordimeform in ovariectomized, steroid-primed female rats. Pharmacol Toxicol 1991;68:131-6.

30. Hooshmandi Z, Rohani AH, Eidi A, Fatahi Z, Golmanesh L, Sahraei $\mathrm{H}$. Reduction of metabolic and behavioral signs of acute stress in male Wistar rats by saffron water extract and its constituent safranal. Pharm Biol 2011;49:947-54.

31. Hemmati M, Asghari S, Zohoori E, Karamian M. Hypoglycemic effects of three Iranian edible plants; jujube, barberry and saffron: correlation with serum adiponectin level. Pak J Pharm Sci 2015;28:2095-9.

32. Dehghan F, Hajiaghaalipour F, Yusof A, Muniandy S, Hosseini SA, Heydari $S$, et al. Saffron with resistance exercise improves diabetic parameters through the GLUT4/AMPK pathway in-vitro and in-vivo. Sci Rep 2016;6:25139.

33. Amin B, Nakhsaz A, Hosseinzadeh H. Evaluation of the antidepressant-like effects of acute and sub-acute administration of crocin and crocetin in mice. Avicenna J Phytomed 2015;5:458-68.

34. Hashimova UF, Kamilova NK, Babaev KF, Shukurova PA, Hasanova SI, Abbasov RY. Effects of saffron (Crocus sativus I. iridaceae) on blood level of follicle-stimulating hormone, and number and dynamics of body weight of offspring in female rats. Adv Gerontol 2017;30:436-41.

35. Groothuis PG, Dassen HH, Romano A, Punyadeera C. Estrogen and the endometrium: lessons learned from gene expression profiling in rodents and human. Hum Reprod Update 2007;13: 405-17.

36. Nelson JF, Felicio LS, Osterburg HH, Finch CE. Altered profiles of estradiol and progesterone associated with prolonged estrous cycles and persistent vaginal cornification in aging C57bl/6J mice. Biol Reprod 1981;24:784-94. 